\title{
Childhood Myelodysplastic Syndrome
}

National Cancer Institute

\section{Source}

National Cancer Institute. Childhood Myelodysplastic Syndrome. NCI Thesaurus. Code C68744.

An uncommon hematologic malignancy occurring during childhood. Many of the morphologic, immunophenotypic, and genetic changes seen in adult myelodysplastic syndromes are also observed in the childhood variants of the disease. Children present with neutropenia and thrombocytopenia more often than adults, and bone marrow hypocellularity is more often seen in children than adults. 